\title{
eJRIEPS
}

Ejournal de la recherche sur l'intervention en éducation physique et sport

$43 \mid 2018$

Varia

\section{Identifier la singularité du rapport au savoir d'un étudiant-stagiaire en éducation physique en situation d'enseignement}

Frédéric Leroy et Ghislain Carlier

\section{OpenEdition}

Journals

Édition électronique

URL : http://journals.openedition.org/ejrieps/483

DOI : $10.4000 /$ ejrieps.483

ISSN : 2105-0821

Éditeur

ELLIADD

Référence électronique

Frédéric Leroy et Ghislain Carlier, « Identifier la singularité du rapport au savoir d'un étudiant-stagiaire en éducation physique en situation d'enseignement », eJRIEPS [En ligne], 43 | 2018, mis en ligne le 01 janvier 2018, consulté le 01 août 2019. URL : http://journals.openedition.org/ejrieps/483 ; DOI :

10.4000/ejrieps.483

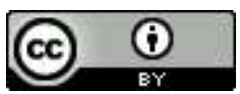

La revue eJRIEPS est mise à disposition selon les termes de la Creative Commons Attribution 4.0 International License. 


\section{eJRIEPS 43 janvier 2018}

\section{Identifier la singularité du rapport au savoir d'un étudiant-stagiaire en éducation physique en situation d'enseignement}

Frédéric Leroy et Ghislain Carlier

Université Catholique de Louvain, Belgique

Résumé

Cette recherche s'inscrit dans le cadre théorique de la didactique clinique. Elle a pour objectif d'identifier les effets de la formation initiale (FI) sur les savoirs enseignés par les étudiants-stagiaires en éducation physique en dernière année et sur leur rapport au savoir. A partir d'une méthodologie temporelle, caractéristique de la didactique clinique, nous présentons une étude de cas, celle d'Annabelle. La recherche révèle que les savoirs enseignés sont, non seulement dictés à l'enseignant par son propre rapport au savoir, dépendant de son histoire, comme il a été maintes fois observés dans d'autres recherches, mais surtout, prescrits par la Fl à travers le maître de stage et le superviseur. Cet assujettissement important, tant à l'école de formation qu'à l'école de stage, est révélateur de tensions constatées au cours des différents entretiens menés avant et après la séance observée in situ. Le rapport au savoir se révèle être un indicateur précieux quant à la logique de formation et à l'identité professionnelle naissante des étudiants.

Mots clés : didactique clinique, savoirs, rapport au savoir, formation initiale, stagiaires, maître de stage

\section{Introduction}

En communauté française de Belgique, la formation initiale $(\mathrm{FI})$ des enseignants, organisée en Haute Ecole (HE), repose sur le principe d'alternance formation-travail par le biais des stages en école répartis durant les trois années de formation. Ils couvrent 480 heures. Selon le décret du 12 décembre 2000 (Communauté française de Belgique, article 15 , p. 5), les stages constituent « des activités pédagogiques pratiques en situation réelle » qui permettent aux étudiants de se familiariser avec le terrain et d'acquérir des savoirsfaire, tout en étant accompagné dans leurs premiers pas par des enseignants expérimentés, les « maîtres de stage » (MS), qui les accueillent dans leur école. 


\section{eJRIEPS 43 janvier 2018}

Les étudiants-stagiaires sont amenés à enseigner des savoirs aux élèves, se plaçant ainsi dans un système didactique articulé sous la forme de deux triangles didactiques imbriqués.

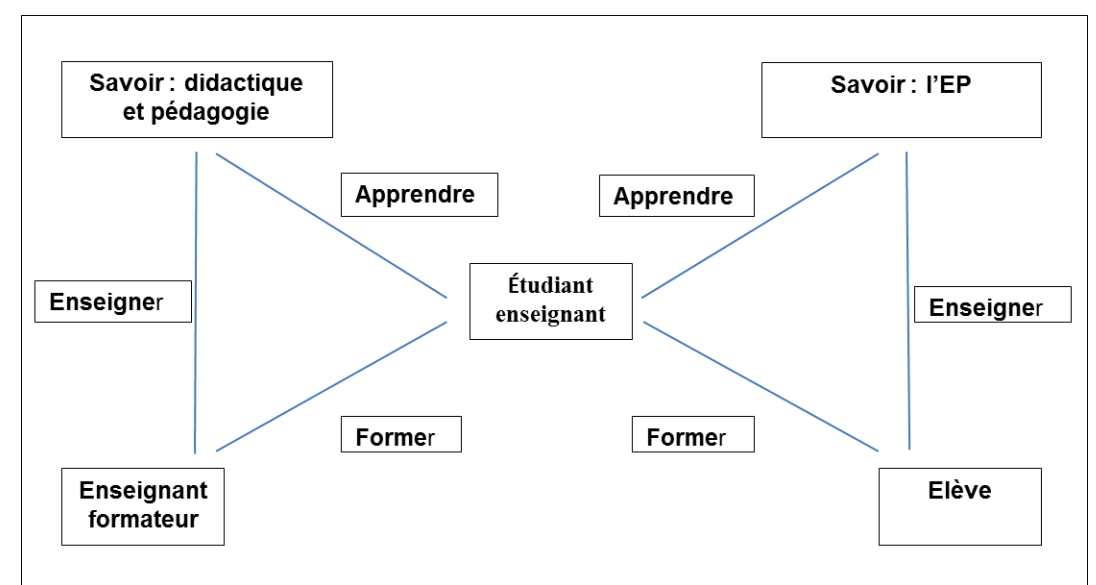

Figure 1. Quadrilatère didactique de la formation initiale d'enseignant (d'après Vanlerberghe, 2007, p. 38).

Inspiré par le modèle de représentation de la situation pédagogique de Houssaye (1988), Vanlerberghe (2007) place l'étudiant au coeur du système dans lequel les relations savoirenseignant-étudiant sont organisées de façon plus spécifique et correspondent mieux à la situation particulière de la formation d'enseignants. II considère que «l'étudiantenseignant » est au centre de la formation : celui-ci est à la fois étudiant, quand il est en situation d'apprentissage et à la fois enseignant, quand il est face aux élèves. Dans cette modélisation, les savoirs enseignés en formation initiale sont donc, à priori, destinés à influencer les savoirs enseignés à l'élève par l'étudiant en situation de stage (étudiantstagiaire). A partir de cette modélisation de l'acte d'enseignement-apprentissage, nous interrogeons, dans notre étude, le pôle du savoir et plus particulièrement la relation entre l'étudiant-enseignant, que nous qualifierons d' «étudiant-stagiaire » et le savoir à enseigner. Ceci nous amène à nous pencher d'avantage sur le concept de rapport au savoir.

\section{Le cadre de la didactique clinique}

Cette étude se situe dans le cadre de la didactique clinique (Terrisse \& Carnus, 2009). Cette approche se caractérise par le croisement « d'une analyse didactique, centrée sur le savoir en jeu dans les interactions didactiques entre le professeur et les élèves à partir des concepts de la recherche en didactique, et une analyse clinique, " au cas par cas ", 


\section{eJRIEPS 43 janvier 2018}

centrée sur l'étude d'un sujet professeur, nécessairement singulier, étudié dans un contexte particulier, sa classe » (Brossais \& Terrisse, 2009, p. 116).

La recherche en didactique clinique valorise "le cas par cas" (Terrisse, 2008), prenant en compte la singularité du sujet et la singularité des pratiques. Cette option oriente la recherche sur les études de cas « puisque le terrain est toujours unique » (Terrisse, 2008, p. 183). De façon à conduire plus efficacement des travaux sur le sujet enseignant, Terrisse et Carnus (2009) développent «une théorie du sujet » reposant sur trois présupposés en interaction: le sujet enseignant est "singulier, assujetti et divisé " (Carnus, 2009).

Le sujet est singulier car il s'organise à partir de trois formes de "déjà-là " décisionnels construits à travers son histoire et ses expériences antérieures. Ces "déjà-là » agissent comme des «filtres" (Loizon, 2009) en rapport avec les pratiques antérieures. On distingue un "déjà-là » conceptuel, en référence aux croyances, valeurs et représentations du sujet; un "déjà-là » intentionnel lié aux intentions didactiques de l'enseignant et un " déjà-là expérientiel, en rapport aux diverses expériences du sujet. Ces «filtres » rendent compte de la part d'inconscient agissant à l'insu du sujet enseignant dans son choix des savoirs à enseigner et de ses interactions.

L'enseignant est assujetti, de façon interne en rapport aux "déjà-là " décisionnels, sources de tensions et conflits intérieurs, mais également de façon externe à différents niveaux. En effet, il est assujetti à une institution, qu'elle soit fédérale, si l'enseignant travaille en club, ou scolaire, ou encore les deux. Selon Loizon (2014), la pratique en club exerce une forte influence sur certaines décisions du professeur d'éducation physique en structurant son « déjà-là » décisionnel.

Enfin, l'enseignant est divisé entre le savoir idéal qu'il souhaite transmettre et le niveau réel des élèves. Il est également divisé entre ce qu'il sait et ce qu'il est " supposé savoir ", entre ce qu'il dit qu'il fait et ce qu'il fait, ce qu'il faut qu'il fasse et qu'il ne peut s'empêcher de faire. "Ce troisième présupposé intègre la dimension de l'inconscient, source de nombreuses divisions chez le sujet enseignant »(Carnus, 2009, p. 69).

La notion de "sujet supposé savoir ", utilisée par Chevallard et empruntée à Lacan (Terrisse, 2010), permet de rendre compte de la place symbolique qu'occupe tout enseignant. Pour les élèves, il est censé savoir mais il sait aussi qu'il ne sait pas tout, ou n'est pas compétent, notamment en début de carrière, face à la multitude des activités physiques qui peuvent être proposées en éducation physique. Dans l'observation des pratiques des enseignants débutants, cela permet de comprendre la recherche de 


\section{eJRIEPS 43 janvier 2018}

« légitimité didactique » car, en tant que stagiaires, leur position symbolique est mal assurée. L'analyse de pratiques révèle des stratégies didactiques créées par des enseignants débutants pour maintenir cette position symbolique.

Dans cette recherche, nous sommes amenés à mobiliser ces différents concepts dans l'observation et l'analyse des pratiques d'enseignement. Ceux-ci, qualifiés par Terrisse d' « analyseurs de pratique » permettent de préciser « le savoir en jeu, ses modifications en cours de séances, son lien avec l'histoire de l'enseignant et son rapport à l'Activité Physique Sportive et Artistique (APSA) utilisée dans son enseignement » (Terrisse, 2016, p. 423).

\section{Le rapport au savoir comme objet d'étude}

La notion de rapport au savoir est abordée dans différentes approches du champ des sciences de l'éducation : clinique (Blanchard-Laville, Beillerot \& Mosconi, 1996) socioanthropologique (Charlot, Bautier \& Rochex, 1992), anthropologique (Chevallard, 1992) et didactique (Maury \& Caillot, 2003). Pour Charlot (1992), le rapport au savoir s'envisage de manière épistémique et identitaire. La dimension épistémique questionne le rapport au savoir dans la nature de l'acte d'apprendre et correspond à la question : « apprendre, c'est avoir quel type d'activité ? ». Le rapport identitaire pose la question du rapport du sujet à lui-même et interroge le «pourquoi apprendre»? Chevallard (1992) propose de distinguer le rapport personnel et le rapport institutionnel à l'objet de connaissance. Le sujet qui entre dans une institution devient «assujetti » à celle-ci. Par conséquent, évoquer la notion de rapport au savoir consiste à parler de la relation qu'un sujet établit avec les savoirs institutionnels ou non institutionnels qu'il a appris ou auxquels il est confronté. Enfin, l'approche didactique propose d'utiliser la locution au pluriel car les savoirs sont multiples. Comme le précise Caillot (2014), on parlera « des savoirs » mais le pluriel vaut aussi pour « rapport » car il n'existe pas un seul type de rapport aux savoirs. «Un enseignant a de multiples rapports aux savoirs : rapports aux savoirs disciplinaires qu'il a appris et qu'il doit faire apprendre, rapport aux savoirs professionnels acquis dans une formation professionnelle ou sur le tas, qui eux-mêmes se déclinent en de nombreux savoirs (savoirs didactiques, savoirs de gestion de classes et de conflits, savoirs administratifs, etc.) 》(Caillot 2014, p. 13). En présentant les résultats de différentes études relatives à l'analyse des pratiques en sports de combat, Terrisse (2010) introduit cette notion en évoquant les raisons particulières qu'a chaque enseignant de choisir et de traiter un savoir : « dans son activité quotidienne, le professeur met l'accent sur tel ou tel 


\section{eJRIEPS 43 janvier 2018}

type de savoir auquel il tient et non sur un autre. Nous émettons alors l'hypothèse, maintes fois observée en recherche, que ce choix est dicté à l'enseignant par son propre rapport au savoir, lui-même dépendant de son histoire de pratiquant ou de celle d'enseignant, voire d'entraîneur » (Terrisse, 2016, p. 4).

Dans le champ de la didactique clinique, les recherches de Jourdan (2009) puis celles de Ben Jomâa et Terrisse (2011) s'intéressent au rapport au savoir en éducation physique. Ils le définissent comme « la façon dont l'enseignant conçoit et traite l'activité qu'il enseigne au moment de l'épreuve de transmission des savoirs " (Ben Jomâa \& Terrisse, 2011, p. 4). II en ressort que les connaissances issues de l'expertise personnelle et corporelle de l'enseignant pèsent significativement sur sa façon d'enseigner et sur ses choix d'enseignement. "Les savoirs ainsi transmis ne sont pas seulement issus de son rapport « officiel » au savoir (...) mais ils ressortent aussi de sa pratique corporelle de spécialiste dans une APS » (Ben Jomâa \& Terrisse, 2011, p. 17).

La spécificité de la discipline " éducation physique » a amené Jourdan (2009) à étudier le rapport au corps des formés, en se focalisant sur l'objet de savoir que sont les activités physiques et sportives, afin de cerner comment s'y révèlent leurs logiques professionnelles. Le rapport aux activités physiques sportives et artistiques (APSA) est envisagé comme point de focus du rapport au savoir des formés et de la spécificité de la discipline EP. "Cerner le rapport aux APSA consiste alors à interroger le rapport (toujours singulier et personnel), qu'entretiennent l'étudiant et le professeur stagiaire à l'APSA en tant qu'objet de savoir » (Jourdan, 2009, p. 36). Pour les étudiants-stagiaires, ces activités physiques sont tout autant de savoirs à acquérir que de savoirs à enseigner ; ce qui nous renvoie au modèle proposé supra par Vanlerberghe (2007). Questionner le rapport au savoir des étudiants en $\mathrm{Fl}$ revient à interroger la $\mathrm{Fl}$ et le rapport des étudiants aux "savoirs professionnels pour aider à comprendre des effets-formation " (Altet, 2000, p. 37). S'intéressant à l'analyse de pratiques en formation et aux effets de celle-ci sur le rapport à la formation et aux savoirs acquis, les travaux d'Altet (2000) ont permis de dégager des catégories du rapport au savoir distinguant un rapport instrumental, professionnel et intellectuel au savoir. Cette classification présente l'intérêt de faire émerger la logique " d'apprentissage de savoirs et de savoir-faire et de leur application " (Altet, 2000, p. 39). Dès lors qu'il est question d'apprentissage et d'enseignement de savoirs, il convient de nous attarder quelque peu sur cette notion du savoir. 


\section{eJRIEPS 43 janvier 2018}

\section{La question des savoirs des enseignants}

Altet (2003) propose une typologie des savoirs des enseignants. Elle distingue les savoirs théoriques, qu'elle scinde en savoirs à enseigner et savoirs pour enseigner, des savoirs pratiques. Les savoirs à enseigner constituent les savoirs académiques, scientifiques et disciplinaires, «didactisés » pour être enseignés aux élèves. Les savoirs pour enseigner sont les savoirs pédagogiques et didactiques destinés à outiller les enseignants dans les différentes disciplines. Quant aux savoirs pratiques, ceux-ci proviennent de l'expérience et peuvent être nuancés en savoir sur la pratique, les savoirs procéduraux, le comment faire, et les savoirs de la pratique, se référant plutôt au savoir y faire, construits à partir des situations vécues. Ces différents savoirs, dans l'action, s'articulent l'un avec l'autre, formant à l'issue de l'expérience nouvelle, des savoirs nouveaux, "savoirs de la pratique qui permettront au sujet de s'adapter à la situation » (Altet, op. cit., p. 35). Cette typologie a le mérite de structurer de façon générale les savoirs. Elle reste néanmoins incomplète pour percevoir, ce qui, en EP, relève des savoirs théoriques, les savoirs à enseigner et les savoirs pour enseigner. Dans sa thèse portant sur la référence de l'enseignant d'éducation physique en karaté, Heuser (2009) distingue différents savoirs à enseigner que nous pensons généralisables à d'autres disciplines de l'EP. II s'agit de savoirs techniques, stratégiques, éthiques, réglementaires et sécuritaires. Quant aux savoirs pour enseigner, ils relèvent de différents modèles d'enseignement ou courants pédagogiques (behavioriste, cognitiviste, constructiviste). Ceux-ci constituent « un modèle général qui s'inspire d'une certaine philosophie de l'éducation et qui va déterminer l'orientation générale d'un processus d'enseignement-apprentissage dans un contexte donné », selon Cloes, Berwart et Frederic (2010, p. 5).

\section{Objectif de recherche}

L'analyse de la pratique effective d'enseignement d'une activité physique et sportive permet de « s'intéresser à la question du savoir qui constitue l'enjeu de la relation entre un professeur et des élèves » et de «comprendre ce qui se joue dans cette relation » (Loizon, Margnes \& Terrisse, 2008, p. 69)

L'objectif de cette étude consiste à identifier, dans un premier temps, les savoirs enseignés par les étudiants-stagiaires en EP en dernière année de $\mathrm{FI}$, et, dans un second temps, les effets de cette FI sur l'enseignement réel des stagiaires et sur leur rapport au savoir. 


\section{eJRIEPS 43 janvier 2018}

\section{Méthodologie}

L'approche clinique implique de centrer l'analyse sur un sujet, singulier. Cela suppose l'inscription de la recherche dans une optique qualitative en utilisant des études de cas (Albarello, 2011) pour comprendre les raisons à l'origine des actes observés. Selon Terrisse (2000) : « la modalité de recueil de données intègre l'évolution temporelle à partir de trois temps de l'interaction didactique, dont l'après-coup est un des éléments fondamentaux, qui donne sens aux deux autres ; la notion d'épreuve qui garantit la prise en compte, par la recherche, du réel de la situation, celle où la validité du savoir est éprouvée (en classe ou dans une situation de confrontation ou de compétition, qui sont souvent les modes d'évaluation en EPS) ».

\section{1. Recueil des données}

La collecte des données s'est effectuée au moyen d'enregistrements audio au cours des différents entretiens ainsi que d'enregistrements vidéo, réalisés in situ lors de la séance enseignée aux élèves. Tout en respectant la méthodologie cohérente par rapport au cadre théorique auquel nous nous référons, nous avons adopté une méthode hybride de recueil de données, originale et inhabituelle, dans la mesure où elle prend inspiration dans deux autres champs de recherche. Ce choix nous est apparu stratégique au regard de notre double posture de formateur-chercheur. En effet, nos préoccupations se sont portées d'une part, sur la volonté, à priori, de limiter les biais inhérents à cette posture et d'autre part, de favoriser la verbalisation en tenant compte des limites que pourraient imposer le fait, pour un étudiant, de s'exprimer face à un formateur avec qui il est encore en contact dans le cadre de sa formation.

5. 1. 1. Entretiens de « déjà-là » réalisés à partir de la «méthode des scénarios 》

La méthode des scénarios (MDS) est une technique d'enquête qualitative peu répandue et mobilisée principalement dans les recherches en communication des organisations. Pour élaborer les scénarios, il est nécessaire de décrire la situation qui va être présentée aux participants de façon à les inciter à réagir, à les stimuler pour qu'ils s'y projettent. Meyer (2008, p. 135) suggère de « construire des scénarios qui déclenchent et provoquent une justification sur certains faits ou comportements du quotidien professionnel des individus ou d'expériences personnelles spécifiques ". II s'agit donc de contextes qui les concernent directement et qui les placent, de façon « artificielle », dans une situation plutôt décalée ou caricaturale permettant d'activer l'imagination et l'intérêt et solliciter la discussion (Julien, Lamonde \& Latouche, 1975). 


\section{eJRIEPS 43 janvier 2018}

Pour le premier scénario proposé, nous cherchons à savoir quels sont les cours marquants de la formation (ceux qui présentent suffisamment d'importance, de sens et de valeur pour être évoqués) et indirectement, ce qui va être utile - et utilisé - dans le métier d'enseignant ainsi que la manière dont les étudiants perçoivent la formation. Dans le second, il s'agit de questionner les représentations qu'ont les étudiants d'eux-mêmes (leur identité professionnelle) et leurs conceptions quant à l'enseignant « idéal ». Indirectement, le rapport au MS et au superviseur ainsi que la place des stages pédagogiques dans la formation sont abordés. Nous renvoyons le lecteur à l'annexe 1 qui présente les deux scénarios proposés sous la forme d'un tableau.

\section{1. 2. Entretien ante séance réalisé à partir de « l'instruction au sosie »}

La démarche d'instruction se déroule en deux phases. La première consiste à répondre à la question suivante : « suppose que je sois ton sosie et que demain je me trouve en situation de te remplacer dans ton travail. Quelles sont les instructions que tu devrais me transmettre afin que personne ne s'avise de la substitution ? » (Clot, 1995, p. 55). La verbalisation provoquée par la mise en situation d'instruire le sosie amène le sujet à dévoiler sa pensée, révélatrice des choix et des dilemmes qui la constituent (Werthe, 1997). Puis, dans la deuxième phase, l'instructeur va se confronter aux résultats de son échange et les commenter par le biais de l'écriture. Dans notre étude, nous mobilisons une forme d'instruction au sosie plus proche de celle d'Oddone et al. (1981) car nous n'avons mis en place que la première phase de l'instruction relative uniquement à la verbalisation de la pratique. Cette représentation que l'étudiant-stagiaire se fait de son activité d'enseignement se verra confrontée à l' "épreuve " par le chercheur lors de la première phase de traitement des données précédant l'entretien d' " après-coup ». Nous considérons qu'il s'agit, en quelque sorte, d'une valeur ajoutée à l'analyse de la pratique ; pratique décrite par le sujet lui-même, guidé par le questionnement du sosie pour « aider l'instructeur à déployer sa pensée et aller au-delà de la première activité » (Mieusset, 2013, pp.171-172).

Cet entretien ante séance amène donc l'étudiant-stagiaire à relater de façon très précise, et anticipativement, la façon dont il va mettre en œuvre sa préparation, la façon dont il va mettre en scène les objets de savoir et comment il compte interagir avec les élèves pour faire évoluer les contenus enseignés. La verbalisation de l'activité de l'étudiant permet, d'une part, de faire émerger les savoirs en jeu au cours de l'épreuve, et d'autre part, de mettre au jour la position subjective du sujet dans l'activité d'enseignement-apprentissage. 


\section{eJRIEPS 43 janvier 2018}

5. 1. 3. L' " épreuve "

Après avoir mené l'entretien ante, nous sommes allés observer la pratique effective d'enseignement. Le temps de "l'épreuve " réside dans des observations de la pratique réelle réalisées in situ soutenue par un enregistrement vidéo visant «à identifier la "mise à l'épreuve" des savoirs détenus par l'enseignant dans la contingence des situations de classe » (Buznic-Bourgeacq, 2009, p. 136).

\section{1. 4. Entretien post séance}

Cet entretien permet de recueillir à chaud, juste après la séance, les appréciations de l'enseignant sur ce qui vient de se passer pour en donner une première description et, éventuellement une justification sur les écarts entre intention et décision, et de revenir sur l'un ou l'autre événement marquant observé au cours de la séance.

5. 1. 5. Entretien d' " après-coup »

Un premier traitement réalisé à partir de la triangulation des données récoltées au cours de l'entretien ante (instruction au sosie et préparation écrite) et au cours de l'épreuve permet au chercheur d'établir des hypothèses interprétatives en vue de préparer l'entretien d'" après-coup ». Réalisé six mois après l'épreuve, alors que le sujet est diplômé, l'entretien d' " après-coup " est construit sur le même mode que la " méthode des scénarios " telle que décrite au point 5.1.1 pour l'entretien de déjà-là. La mise en situation, à travers le scénario proposé, permet au sujet de reconstruire sa position à partir des traces mnésiques conservées. II s'agit d'identifier le point de vue du sujet sur sa propre pratique et sur sa formation. L'intérêt principal relève de l'interprétation en questionnant les hypothèses du chercheur au regard de la logique singulière du sujet. Dans le cas présent, le concept d' "après-coup " prend d'autant plus de sens que l' étudiant a quitté la haute école et que le questionnement sur " l'après-FI 》 est engagé dans une mise à distance aussi bien temporelle que spatiale. Nous proposons au lecteur de consulter l'annexe 2 afin de prendre connaissance des deux scénarios proposés pour cet entretien. 


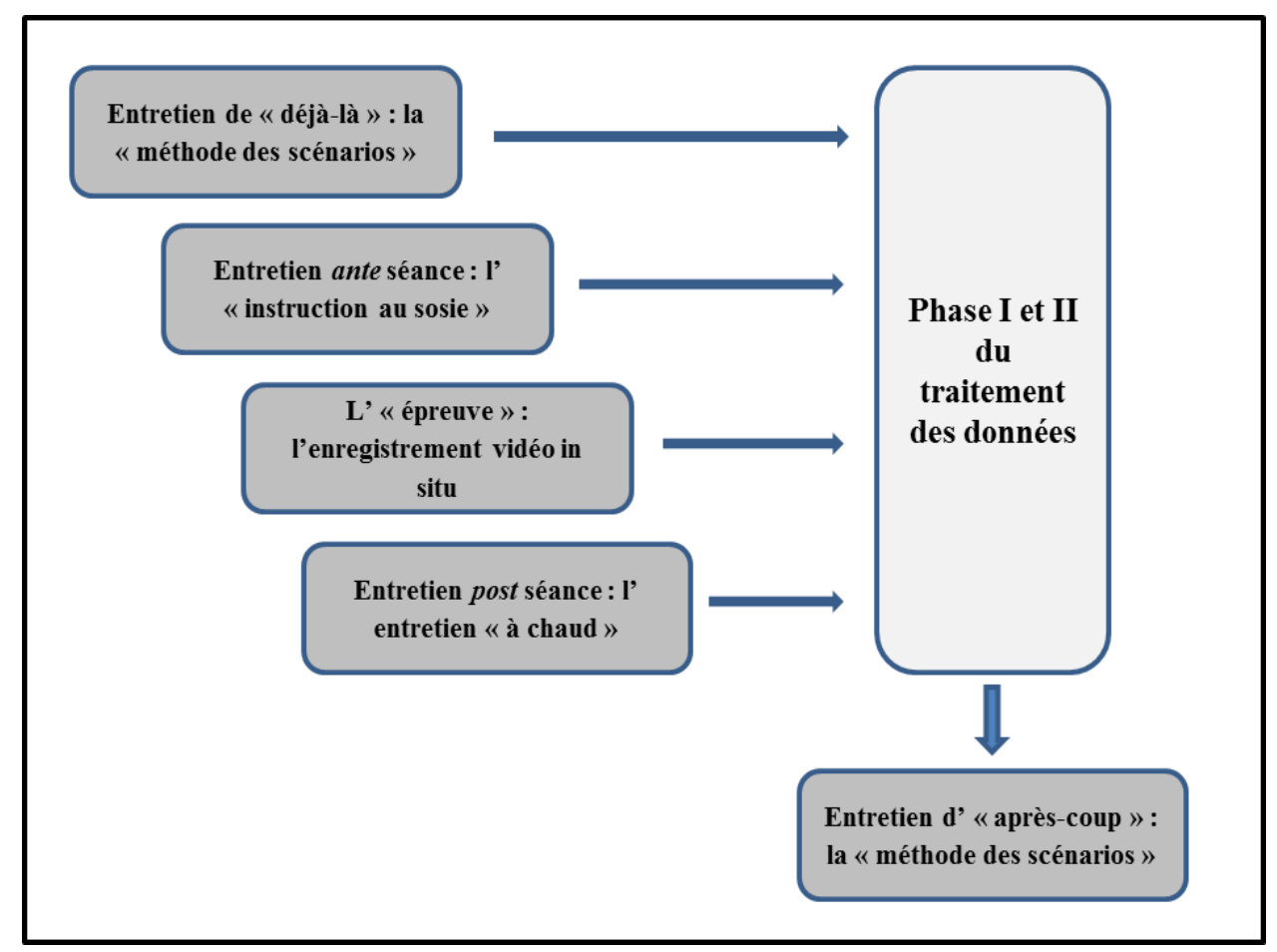

Figure 2. Schéma récapitulatif du protocole de recueil des données.

5. 2. Méthodologie du traitement des données

Le traitement des données comporte trois phases:

- la première phase consiste en une analyse de contenu des entretiens de " déjà-là ", ante séance et post séance.

- Au cours de la deuxième phase, nous analysons le temps de "l'épreuve " en triangulant les données recueillies grâce à l'entretien ante séance, la préparation écrite de la séance réalisée par l'étudiant et le visionnage de la vidéo de la séance.

- La troisième phase est consacrée à l'analyse de contenu de l'entretien d' « aprèscoup ».

Pour interroger le corpus et en retirer les informations nécessaires à notre recherche, nous réalisons une analyse de contenu rigoureuse selon une méthode logico-sémantique (Mucchielli, 1991). Dès lors, la première étape du traitement des données s'oriente vers la création des catégories, de façon à pouvoir coder les unités de sens du corpus. Cette phase se déroule selon une démarche déductive à partir des objectifs de la recherche. L'analyse textuelle est réalisée à l'aide du logiciel NVivo reposant sur une approche réflexive du chercheur au sens de Lejeune (2008), c'est-à-dire sans automatisation de la démarche et mobilisant davantage une approche thématique. A partir des concepts théoriques évoqués ci-dessus, trois grilles de codage sont créées, en lien avec les quatre entretiens réalisés. L'utilisation du logiciel NVivo demande à l'analyste de créer des « 


\section{eJRIEPS 43 janvier 2018}

nœuds " correspondant aux catégories construites et de lire le corpus afin de marquer, comme à l'aide d'un surligneur, et de coder les éléments textuels significatifs.

Nous avons raisonné à partir de cinq regroupements en lien avec les savoirs et le rapport au savoir ainsi qu'avec les concepts cliniques identifiés dans le cadre théorique.

Tableau I. Tableau récapitulatif des catégories de codage des verbatims.

Catégorie A : type de rapport au savoir chez les étudiants en formation (selon Altet 2000)

1) un rapport instrumental aux savoirs

2) un rapport professionnel au savoir

3) un rapport intellectuel au savoir

Catégorie B : la nature des savoirs (selon Altet, 2003)

1) les savoirs théoriques, parmi lesquels on distingue les savoirs à enseigner ( $\mathrm{SaE}$ ), et les savoirs pour enseigner (SpE)

2) les savoirs pratiques, parmi lesquels on distingue les savoirs sur la pratique (SsP) et les savoirs de la pratique (SdP)

Catégorie C : type de SaE en éducation physique (selon Heuser)

1) des savoirs techniques

2) des savoirs stratégiques

3) des savoirs éthiques

4) des savoirs règlementaires

5) des savoirs sécuritaires

Catégorie D : type de SpE (basé sur l'étude de Cloes, Berwart et Frédéric, 2010)

1) le courant behavioriste

2) le courant cognitiviste

3) le courant constructiviste

4) le courant humaniste

Catégorie $\mathrm{E}$ : présupposés à la base de la « théorie du sujet enseignant » (Terrisse et Carnus, 2009)

1) le sujet enseignant est singulier à partir de ses « déjà-là » décisionnels

2) le sujet enseignant est assujetti

3) le sujet enseignant est divisé

4) le sujet enseignant est supposé savoir (position symbolique). 


\section{eJRIEPS 43 janvier 2018}

\section{Etudes de cas : Annabelle}

Cette étape de la construction du cas d'Annabelle montre au lecteur l'articulation des différents « analyseurs de pratique », repris comme catégories de codage des entretiens, de façon à dégager sa logique singulière de sujet enseignant. La construction du cas se fera par une description des résultats qui présentent, ce qui, dans le discours du sujet, permet de répondre à nos objectifs de recherche. Cette présentation permet de croiser les savoirs avec la logique singulière du sujet en mettant en évidence les préoccupations qui l'animent. Pour cette étude, nous ne développerons que les savoirs majoritairement mis en évidence que sont les savoirs techniques, soit la catégorie de codage $\mathrm{C} 1$.

6. 1. Présentation de l'étudiante-stagiairelnscrite à la base pour des études de kinésithérapie, Annabelle s'est réorientée vers l'EP après avoir recommencé sa première année sans succès. Pratiquante de rugby et de tennis de table, elle a également une expérience d'encadrement de jeunes dans des mouvements de jeunesse et via des cours de tennis et de tennis de table lors de stages de vacances. L'étude de cas réalisée avec Annabelle repose sur une séance d'EP d'une durée de 100 min qu'elle a enseignée à des élèves de deuxième année du secondaire. Cette séance était scindée en deux et, suivant les consignes de la MS, abordait deux disciplines sportives différentes : l'athlétisme et le tennis de table. La leçon d'athlétisme avait pour objet de savoir l'apprentissage du sprint et celle de tennis de table, l'apprentissage du revers puis du coup droit.

\section{2. Les savoirs enseignés}

6. 2. 1. Son déjà-là expérientiel : l'importance du vécu dans l'APS

Le vécu personnel entre pour une large part dans la façon dont Annabelle aborde ses séances de sprint et de tennis de table. C'est au cours de l'entretien post-séance qu'elle nous confie l'impact de son expérience de joueuse de tennis de table sur son enseignement : " l'histoire de la porte qu'on ouvre, on trouve jamais ça dans les livres mais c'est simplement mon entraineur qui m'a appris cette image-là quand j'étais petite». Cet apprentissage par l'utilisation de métaphores a bien fonctionné pour elle, ce qui la conduit à le transposer dans son enseignement en constatant que «ça fonctionne bien » pour aider à apprendre le mouvement du revers. Pour Annabelle, ce n'est pas possible de « connaitre des images à donner pour les gestes techniques » sans avoir jamais donné cours de tennis de table. Le fait d'entrainer les petits au club l'a amenée à réfléchir et trouver des consignes plus imagées, « des repères », de façon à faire comprendre et faire progresser. 


\section{eJRIEPS 43 janvier 2018}

A propos de la séance de sprint, Annabelle puise dans son vécu de débutante en sprint, quand, au cours de la formation, elle a éprouvé des difficultés à performer en athlétisme. Dès lors, l'abondance des consignes données aux élèves font écho avec le besoin qu'elle a connu d'être bien suivie et aidée par la formatrice : «si on m'expliquait pas, j'avais tout le temps la jambe arrière beaucoup trop loin (...) j'arrivais vraiment pas à partir et donc je pense que ça m'a frustrée ". Cette situation d'échec vécue l'amène donc à enseigner en apportant beaucoup d'explications, de précisions à ses élèves pour que «ça les aide », désirant éviter par-là, de faire vivre aux élèves l'expérience frustrante de ne pas y arriver.

6. 2. 2. Le SaE : l'incorporation du déjà-là expérientiel, du déjà-là intentionnel et du SdP

Les objectifs de la séance de sprint, décrits au cours de l'entretien ante séance, consistent à enseigner la technique de départ ainsi qu'améliorer la technique de course. Elle insiste, lors des éducatifs de course, sur l'action du genou, il s'agit d' " apprendre à travailler avec le genou haut et loin devant ". Ayant déjà enseigné cette discipline lors d'un stage précédent, Annabelle considère cette première expérience comme une référence sur laquelle elle s'appuie pour relever quelques défauts dans la réalisation des exercices de courses : « elles pensent un peu trop à la gymnastique (...) donc c'est souvent leur défaut ; donc bien insister sur la jambe avant qui doit être fléchie ». Elle aborde ensuite la technique du départ et amène, dans un premier temps, les élèves à déterminer leur pied d'impulsion. Elle enchaine ensuite avec l'apprentissage du départ au sol pour lequel elle passe beaucoup de temps sur l'explication et la démonstration des différentes positions : « je leur décortique, je leur explique la technique de départ et je leur montre comment elles doivent se positionner ». De nouveau, au cours de cette partie principale de la séance, elle se base sur son stage précédent pour corriger les erreurs repérées chez les élèves. Cette intervention visant l'amélioration du geste technique est vécue avec plus d'aisance pour Annabelle qui constate que, même si elle n'a pas beaucoup d'expérience, pour cette leçon, elle « repère plus facilement les défauts".

L'objectif poursuivi au cours de la deuxième période réside dans l'enseignement du revers en tennis de table. Dans un premier temps, Annabelle propose une situation visant le travail du déplacement suite au bilan effectué au cours précédent quant à l'attitude trop statique des élèves : "le tennis de table, si j'oblige pas des déplacements, elles restent à la table, complètement figées, elles bougent pas ». S'appuyant à nouveau sur son vécu de stage, «filtré » par son expérience et son regard de joueuse et d'entraineur, elle ajuste sa deuxième séance. 


\section{eJRIEPS 43 janvier 2018}

6. 2. 3. Les $\mathrm{SpE}$ : un modèle pédagogique prégnant

En lien direct avec les SaE qui, comme nous venons de les décrire sont exclusivement des savoirs techniques, nous observons, lors de l'entretien ante, que les SpE mobilisés sont basés sur le modèle pédagogique behavioriste. Annabelle, au cours des deux périodes d'enseignement, propose des gestes techniques que les élèves doivent reproduire. Son enseignement repose sur des explications verbales et sur des démonstrations des gestes à réaliser, "c'est moi qui montre la technique de départ », pour lequel des critères de réussite sont énoncés, « je rappelle seulement deux, trois critères, élévation rapide des genoux, regard devant, garder le tronc droit ». Ses interventions didactiques se bornent à orienter les élèves pour les amener à la réussite des gestes proposés : "si par exemple elles font un mauvais skipping, je remontre et je corrige, bein, elles doivent le refaire pour essayer d'elles-mêmes corriger pour que ce soit bon la fois d'après ».

\section{3. Effets de la formation sur l'enseignement de l'étudiant-stagiaire}

\section{3. 1. Une position en « tension»}

Au cours de l'entretien post séance, Annabelle fait le bilan de sa séance et se trouve finalement « divisée » entre la nécessité de suivre ce qui est prévu dans la leçon « je voulais quand même prendre le temps de faire les étirements » et de faire évoluer les savoirs " j'ai eu moins le temps de faire mes exercices et mes exercices de remédiation ». Cela se traduit par une situation inconfortable, un constat de non-satisfaction de la leçon « j'ai l'impression de ne pas avoir eu le temps de bien leur expliquer ». De façon générale, Annabelle se trouve en école de stage, comme en école de formation, en « tension », partagée entre la conception de l'enseignement des APS liée à la technique et la performance, et son déjà-là conceptuel, comme nous le verrons au point 6.4.2. En effet, celui-ci se veut davantage axé sur l'amélioration de la motricité des élèves et sur la volonté de susciter l'envie de poursuivre une pratique en dehors de l'école : « on apprend aux élèves à courir vite pour réussir à faire un sprint (...) ce qui est important, c'est d'apprendre à un élève à courir correctement, à savoir courir, pas sur les talons, parce que sinon, il risque d'avoir des problèmes plus tard, c'est plutôt ça qui est important ».

Les objectifs visés par la MS sont de l'ordre de l'apprentissage de gestes techniques et cette approche divise Annabelle qui se rend bien compte qu'elle ne va pas « susciter des intérêts » en enseignant de la sorte. 


\section{eJRIEPS 43 janvier 2018}

6. 3. 2 Une position « assujettie » à l'institut de formation

Malgré le constat d'une MS très assujettissante, Annabelle reconnait s'être améliorée notamment au niveau de l'organisation pratique de ses leçons, même pour le tennis de table. En effet, elle a acquis des SsP grâce aux nombreuses interventions de sa MS. De façon générale, il s'agit de "petites choses qui facilitent la leçon », " des petits trucs qu'elle a déjà testé et qui fonctionnement bien avec ses élèves », ou encore « des petits conseils d'organisation qui font que c'est plus facile ». A propos des intentions didactiques des séances, celles-ci sont déterminées par sa MS en lien avec l'apprentissage de gestes techniques car selon elle, « elles (les élèves) n'avaient pas la technique et donc il faut passer par la technique ». Ce qui conduit donc Annabelle à mettre l'accent sur ces objectifs et à trouver « des stratégies » pour garantir le respect du contrat didactique : « je suis obligée de voir les techniques et je leur promets, on va dire entre guillemets, un match à la dernière leçon pour les stimuler et pour les encourager à apprendre ces techniques ».

Les autres expériences de stage vécues ne lui ont pas permis de pouvoir tenter d'enseigner selon ses propres conceptions : "j'essayerais de voir des mouvements gymniques, des compétences de la gym, sauter, se réceptionner, rouler (...) j'ai jamais pu faire en stage parce que les MS font ça rarement. Quand ils font gym, ils font gym ». Cette approche techniciste est renforcée par la formation initiale au cours de laquelle l'apprentissage technique est prépondérant au sein des cours d'APS et de didactique des APS : « on insiste fort sur la technique, moi j'ai l'impression d'avoir vraiment été influencée fort sur la technique ». Ce constat débouche, au final, sur la conclusion d'Annabelle qu'enseigner les APS ne passe que par l'apprentissage de gestes techniques « souvent, on pense qu'apprendre, c'est juste technique ; s'il n'y a pas de technique, on leur a rien appris ».

Ce focus mis sur l'approche techniciste, comme le décrit Annabelle, est perçue comme une obligation de la part de l'école de formation et les écoles de stage, " pour les profs, pour les superviseurs et les MS, apprendre c'est technique ». Dès lors, face à cette nécessité d'alignement didactique, ou d'isomorphisme, l'innovation se veut timide voire complètement inhibée : «personne n'est assez franc en stage en deuxième, en troisième pour tester quelque chose de complétement nouveau et puis, de, je vais pas dire affronter, mais de pouvoir défendre cette manière d'apprendre ». Ce " non dire " d'Annabelle est révélateur de l'assujettissement prégnant à l'institut de formation et de la place, toute légitime, accordée à la réussite des études. Elle regrette de ne pas avoir pu tenter au 


\section{eJRIEPS 43 janvier 2018}

cours de ses stages de fonctionner à partir des situations qu'elles avaient préparées. Elle se voit contrainte d'attendre d'être diplômée pour pouvoir fonctionner selon " ses idées et des façons de faire » : "à mon avis quand je vais aller enseigner l'année prochaine, je vais l'essayer".

6. 4. Effets de la formation sur le rapport au savoir de l'étudiant-stagiaire

6. 4. 1. Rapport aux savoirs et "Déjà-là » de la FI

Annabelle se positionne principalement dans un rapport au savoir professionnel, dans la mesure où elle accorde beaucoup d'importance aux cours suivis en formation initiale, permettant d'allier théorie et pratique, de façon à " comprendre les différentes manières d'enseigner ». Selon elle, ils traitent de l'enseignement des APS en fonction du niveau et de l'âge des élèves : " en troisième le cours de sports collectifs, c'était lié à l'école et comment je vais aborder avec les élèves le tchouck-ball, comment... et ça c'était intéressant (...) on faisait une demi-heure de didactique, et juste après on allait mettre en application sur le terrain ce qu'on venait de voir ». Par ailleurs, elle regrette le manque de liens entre les cours théoriques et les cours pratiques, l'inverse étant également vrai pour certaines disciplines : " par exemple, on faisait du sprint, on faisait des éducatifs, on ne les apprenait pas dans le but après d'aller les enseigner, on les apprenait parce qu'on devait s'améliorer en sprint ». Du fait de la complexité de trouver des liens entre les cours théoriques et la pratique, qu'il s'agisse de pratique sportive ou de pratique liée à l'enseignement sur le terrain, Annabelle considère finalement que les stages ont joué le rôle de révélateur de cette (non-)articulation.

6. 4. 2. Un déjà-là conceptuel en marge des intentions didactiques

Au cours de l'entretien de déjà-là, Annabelle nous fait également part de sa conception du bon enseignant en EP qui, selon elle, est celui qui parvient à susciter l'intérêt pour les APS par la découverte de celles-ci et à donner le goût de la pratique sportive. Même si le cours d'EP peut ambitionner de séduire les jeunes pour une pratique sportive plus assidue, il s'agit de s'en distinguer au niveau de la démarche didactique proposée. En effet, l'enseignement de l'EP se veut davantage axé vers une approche globale et ludique ; il s'agit de "donner l'envie de jouer et, par le jeu, ça peut susciter leur intérêt je pense ». Ce n'est pas à travers une approche "technique " que l'on va motiver les élèves, confie Annabelle. Pour devenir cette enseignante-là, le « bon prof d'EP », Annabelle pense que la $\mathrm{Fl}$ « met sur la voie » mais " qu'on ne l'est pas en sortant de l'école » ni même en début de carrière car " on reproduit ce qu'on a appris à l'école ", ce qui ne garantit pas le fait d'être un «bon prof ». C'est seulement après avoir acquis de l'expérience, après avoir pu 


\section{eJRIEPS 43 janvier 2018}

tester des choses qu'elle pense pouvoir « trouver comment enseigner aux élèves à faire plein de choses (...), leur donner goût ».

\section{5. L'après-coup de la FI et de l'épreuve}

\section{5. 1. L'après FI : une formation « prête-à-l'emploi »}

Quand Annabelle parle de ses études dans l'entretien d'après coup, elle fait état d'une formation dont le but est de former des enseignants. Faisant référence à son premier intérim dans l'enseignement, Annabelle reconnait l'avoir vécu avec une certaine aisance, possédant tous les outils en mains «pour pouvoir enseigner». Elle présente une Fl qui, pour l'étudiant qui s'y engage, l'amène « à être prêt à enseigner quand il sort », bien qu'elle considère que cette qualité attribuable à la formation ne soit pas une condition unique de professionnalité : “ c'est sûr qu'il faut à côté continuer à se former et continuer à apprendre parce qu'on n'est pas encore calé dans tout au niveau de l'enseignement ». Pour Annabelle, le développement professionnel est une démarche qui fait partie intégrante de la vie de l'enseignant débutant car, même si les différents stages et les cours ont permis de se construire professionnellement, "c'est pas totalement défini en sortant de l'école ».

\section{5. 2. L'après coup de l'épreuve : un assujettissement marquant}

S'il lui fallait proposer à un collègue sa leçon d'athlétisme ou celle de tennis de table, Annabelle est catégorique : « je ne lui donnerais pas celle-là »! En effet, comme nous l'avions constaté au cours des entretiens de déjà-là et post séance, l'approche techniciste n'est pas celle qui correspond à ses conceptions et ses intentions didactiques : « il y a quand même moyen de faire de exercices plus rigolos que juste coup droit, renvoyer, revers, renvoyer ». Annabelle voit sa conception renforcée quand, lors de son premier intérim, elle est amenée à enseigner dans une école technique et professionnelle dans laquelle le cours d'EP est considéré comme le parent pauvre ; il n'y a pas de salle ni de matériel : «c'est un peu la garderie quoi ». Dès lors, pour motiver les élèves à participer à ses cours d'endurance, elle propose des situations « ludiques » comme des courses d'orientation ou encore « un genre de cache-cache ». Elle en a conclu que les élèves avaient apprécié mais surtout qu'avec « ce public-là, si on leur parle de technique pendant une demi-heure, pfff, la fois d'après, c'est fini, on en a plus ».

L'assujettissement à la MS a été relevé de façon marquante au cours des trois entretiens précédents. Poursuivant l'analyse du rapport entre Annabelle et ses MS, l'après-coup apporte des informations complémentaires qui permettent d'approfondir l'étude de cette relation. En débutant son stage, Annabelle s'est décidée à suivre les pas de sa MS au 


\section{eJRIEPS 43 janvier 2018}

point de « se mettre un peu dans le moule » ; «j'essayais de me mettre à deux cent pour cent pour lui montrer que je faisais comme elle ». A la fin de chaque leçon Annabelle vivait un débriefing très détaillé, au cours duquel madame $\mathrm{W}$ lui faisait part de toutes les remarques et observations au sujet de sa leçon. De façon à modifier ses actions selon le feedback reçu et à « faire comme elle, elle faisait », Annabelle était constamment concentrée au cours de ses leçons sachant que madame W prenait note de tous ses faits et gestes : «j'étais tout le temps attentive à deux cents pour cent (...) ça me fatiguait à un point, heu, j'avais du mal le soir ». Annabelle nous confie qu'elle " jouait un rôle » et que l'écart entre les intentions didactiques de sa MS et les siennes révèle d'autres intentions plus profondes « de vouloir combler ses attentes ». S'expliquant à ce sujet, elle dévoile une certaine « séduction » exercée à son insu et liée à cet assujettissement fort : “ c'est bizarre à dire mais, on essaye de faire plaisir à son MS et de montrer vraiment ce que lui a envie de voir et pas ce qu'on est vraiment, enfin, en tout cas, c'est comme ça que je le perçois maintenant». Elle ajoute encore : « je pense que je l'ai fait automatiquement pour la satisfaire, on va dire (...) c'était surtout pour lui faire plaisir, pour être vraiment parfaite on va dire, enfin, essayer en tout cas ». Le processus de transfert, issu du registre de la psychanalyse, apparaît ici de façon forte et bien identifiée par Annabelle, malgré elle. Cette anecdote met en évidence l'existence de phénomènes transférentiels dans l'activité éducative comme déjà décrit par Freud et nombre d'auteurs après lui s'étant penchés sur cette question. Dans le cas présent, nous avançons, à la suite de Filloux (1989, p. 74) que «le transfert est ici tout bonnement assimilé à cette pulsion de domination qu'Adler substitue aux pulsions sexuelles et à la théorie de la libido. Il ne s'agit plus d'un désir amoureux objectal avec ses dimensions de passivité et d'activité, mais bien d'un désir actif d'agression et d'emprise sur l'autre ». En effet, la démarche de transfert d'Annabelle sur sa MS réside en une sorte de «manœuvre de l'autre », visant à « accaparer son affection » en vue d' « imposer une certaine forme de relation » (Filloux, ibid.). Nous faisons l'hypothèse d'une recherche de reconnaissance privilégiée, d'une reconnaissance de ses compétences professionnelles.

La préparation écrite demandée aux stagiaires se révèle dans l'après-coup comme un second assujettissement important. En effet, le fait de devoir atteindre l'objectif prévu initialement dans la préparation place Annabelle en situation de tension : « devoir tout faire pour arriver à l'objectif qu'on avait décidé en début de cycle, c'était quand même un truc stressant quoi ». Elle ne s'autorise pas à sortir de sa préparation écrite, ce qui 


\section{eJRIEPS 43 janvier 2018}

explique l'absence d'écart entre le SaE et le SrE (savoir réellement enseigné) ; Annabelle en parle comme étant « limitée par une préparation qu’on doit suivre ».

\section{Discussion}

L'objectif de notre étude, rappelons-le, réside dans l'identification, d'une part, des savoirs enseignés par Annabelle, et d'autre part, des effets de la FI sur son enseignement réel et sur son rapport au savoir. Les savoirs enseignés ont été mis en évidence au cours de la construction du cas d'Annabelle. Nous proposons maintenant de discuter le cas sous deux angles : celui du rapport au savoir dans ses dimensions épistémique et identitaire selon Charlot (2002), celui du rapport institutionnel au savoir selon Chevallard (1992). L'articulation de cette discussion nous permet ainsi d'apporter un éclairage, dans un premier temps, sur le rapport au savoir de la formation, c'est-à-dire le rapport aux contenus et à leur utilisation. Dans un second temps, les représentations de la formation et du métier sont mises en évidence, en lien avec l'histoire et les conceptions du sujet. Enfin, le rapport institutionnel caractérise l'influence marquante de l'institut de formation sur l'enseignement réel d'Annabelle.

\section{1. Un rapport épistémique au savoir}

Pour Annabelle, apprendre au cours de sa formation signifie allier davantage théorie et pratique, de façon à donner plus de sens aux cours théoriques et amorcer une démarche réflexive dans les cours pratiques (cours d'APS). Elle s'inscrit par-là dans un rapport au savoir de type professionnel comme définit par Altet (2000, p. 38) : "le savoir a une fonction, il permet une distanciation, un recul et favorise un autre regard sur les pratiques (...) ». Ce souhait rejoint le constat, déjà relevé par de nombreux étudiants-stagiaires, que les savoirs théoriques ne servent pas d'appui pour l'action professionnelle et que la formation pourrait dépasser cette difficulté en combinant " les dynamiques de formation ascendantes (à partir de l'action) et descendantes (à partir des savoirs) (Goigoux, Ria \& Toczek-Capelle, 2009, p. 36)». Dans l'entretien d'après-coup, Annabelle maintient son opinion quant à l'importance des cours d'APS et de didactique des APS et elle évoque une formation " prête-à-l'emploi » au sortir des études. Cependant, même si la formation donne les clés pour entrer dans le métier, Annabelle évoque avec lucidité l'importance de poursuivre son développement professionnel grâce aux formations continues car " c'est pas totalement défini en sortant de l'école ". Cette dynamique de développement qu'elle a entamée en formation l'a conduite à prendre conscience de la distance entre le vécu de terrain, les discours tenus par l'école de formation et son déjà-là, l'amenant à construire et 


\section{eJRIEPS 43 janvier 2018}

faire évoluer son identité professionnelle. Pour Wittorski et Briquet-Duhazé (2009, p. 54) « le développement professionnel consisterait alors à accepter cette tension permanente qui serait vécue non pas comme une contradiction mais comme un défi continuellement en mouvance (on ne peut pas apprendre définitivement le métier d'enseignant)». Cette distanciation par rapport à la formation est révélée dans l'entretien d'après-coup par l'écart entre le faire "prescriptif » des stages et de la formation, et le faire actuel, adapté aux caractéristiques du terrain et d'avantage en accord avec son déjà-là .

Les $\mathrm{SaE}$ acquis en FI grâce aux cours d'APS et de didactique des APS, sont orientés vers l'apprentissage de gestes techniques qui paraissent nécessaires pour maîtriser la base des disciplines enseignées. Les $\mathrm{SpE}$, orientés vers un modèle pédagogique de type « behavioriste " sont en lien avec les $\mathrm{SaE}$; il s'agit de reproduire les gestes enseignés et les interactions didactiques sont relatives à l'application de ce modèle. L'étude de Cloes, Berwart et Frédéric (2010) montre que les stratégies d'enseignement des enseignants en EP en Belgique francophone sont considérées comme traditionnelles, basées sur une approche " techniciste ». Ils démontrent une connaissance théorique limitée de l'approche pédagogique socioconstructiviste et les auteurs avancent l'explication d'un manque d'intérêt ou d'une certaine résistance au changement. D'autres résultats de l'enquête mettent en avant l'idée que " les enseignants ne savent pas appliquer cette stratégie d'enseignement dans leur cours ou bien qu'ils travaillent depuis plusieurs années d'une façon qui fonctionne très bien à leurs yeux et qu'ils éprouvent donc quelques réticences à changer de méthode " (Cloes, Berwart et Frédéric, 2010, p. 18). Dans ce contexte, nous pouvons estimer que les $\mathrm{SaE}$ et les $\mathrm{SpE}$ mobilisés par Annabelle font référence à cette doxa ou cette approche communément reconnue qui émerge tant à l'école de formation qu'à l'école de stage. De plus, dans l'après-coup, Annabelle reconnait la mise en œuvre de ce modèle comme plus étant plus facile mais reste ouverte à un autre modèle pédagogique, notamment socioconstructiviste, à partir du moment où, selon elle, on connait ses élèves, par crainte de ne pouvoir gérer sa classe. En effet, la logique de la conduite de la classe devance celle des savoirs enseignés chez les débutants, car la priorité est d'abord celle de garder la classe en activité, de « tenir sa classe » et de « faire autorité » comme le soulignent Goigoux, Ria et Toczek-Capelle (2009).

\section{2. Un rapport identitaire au savoir}

Pour Annabelle, devenir un " bon prof d'EP », c'est être cette enseignante qui suscite l'intérêt pour les APS, qui donne le goût de la pratique sportive ou encore qui enseigne un savoir qui peut avoir une utilité concrète dans la vie de tous les jours. Cette conception 


\section{eJRIEPS 43 janvier 2018}

rejoint une croyance répandue dans la profession qui consiste, par la polyvalence des APS proposées, à permettre aux élèves de découvrir celles qu'ils souhaiteraient pratiquer. Delignières et Garsaux (2004, p. 170) postulent le lien entre la multiplicité des activités présentées et l'orientation sélective, proposant par-là « une éducation physique qui jouerait le rôle d'entremetteur entre les élèves et les activités sportives ». A travers ce rapport identitaire, on prend la mesure de cette appartenance sociale du sujet au système scolaire, permettant de comprendre l'influence de celui-ci par la prise en compte de la dimension sociale qui donne une forme particulière au rapport identitaire au savoir. La première expérience d'enseignement comme intérimaire qu'Annabelle commente lors de l'après-coup, confirme le décalage entre l'approche proposée en formation et ses conceptions de l'enseignement de l'EP. La FI met sur la voie. Mais c'est l'expérience qui permettra de devenir cette bonne enseignante, après avoir pu tester différentes approches, nous dit-elle. Nous comprenons cette assertion comme la preuve de sa « division identitaire ", déjà relevée en entretien post séance quand elle se projetait en tant que future enseignante qui ferait « plus tard (...) plus de situations de jeu (...) beaucoup plus amusantes (...) » en totale correspondance avec son déjà-là conceptuel. Ce n'est qu'après l'école qu'elle deviendra cette « bonne » enseignante, celle qui correspond à son identité professionnelle, celle qui s'est développée en cours de formation mais qui n'est pas encore « aboutie », qui est toujours en construction car la FI « met sur la voie » mais « on ne l'est pas en sortant de l'école » ni même en début de carrière car « on reproduit ce qu'on a appris à l'école ». Comme l'exprime Charlot (op. cit., p. 85), « il est bien des façons de "devenir quelqu'un", à travers les diverses figures de l'apprendre ; mais la société moderne tend à imposer la figure du savoir-objet (de la réussite scolaire) comme passage obligé pour avoir le droit d'être "quelqu'un" ». Dès lors, identifier les divisions existant dans l'acte d'enseignement-apprentissage permet de mieux cerner le profil identitaire de l'étudiant pour l'aider, d'une part, à devenir ce « quelqu'un », cet « enseignant idéal », celui qui est en accord avec son déjà-là, et d'autre part, à traverser cette crise identitaire professionnelle. En effet, à l'instar « des adolescents devant tout à coup faire face à l'inconnu de leur corps transformé, les professeurs stagiaires sont confrontés à une situation d'"inquiétante étrangeté" ॥ (Bossard, 2009, p. 68) générant une grande angoisse existentielle

\section{3. Un rapport institutionnel}

Chez Annabelle, nous pouvons distinguer un assujettissement important à l'école de formation et à l'école de stage à travers la préparation écrite des leçons, source de 


\section{eJRIEPS 43 janvier 2018}

divisions. En effet, l'école de formation impose un format de préparation écrite dans laquelle des objectifs opérationnels doivent préciser les intentions didactiques pour la leçon donnée d'une part, et le déroulement de la séance doit exposer les savoirs à enseigner, d'autre part. Ce document constitue le support de référence tant pour l'étudiant que pour le MS et le superviseur. II rassure l'étudiant car le fait de préparer, c'est anticiper l'organisation, c'est faire en sorte que "ça roule ». C'est ce que Saujat et Serres (2015) qualifient de « genre débutant » ou plutôt d' « usage débutant du genre professionnel enseignant » pour parler des préoccupations qui animent les enseignants novices. Ces préoccupations communes visent à « anticiper leurs difficultés à anticiper et de contenir les effets déstabilisants des inattendus auxquels ne cesse de les confronter ce milieu, en multipliant les attendus » (Saujat \& Serres, op. cit., p. 13). Comme nous le révèle Annabelle dans l'après-coup quand on la questionne sur la quasi correspondance totale entre l'entretien ante et l'épreuve, le fait d'avoir réfléchi préalablement à tout, de n'avoir rien laissé au hasard se révèle être un moyen de ne pas perdre du temps, de pas faire d'erreurs, d'être sûr de ce qu'elle allait dire ; il s'agit véritablement pour elle d'étudier par cœur sa préparation car en stage, les erreurs ne peuvent pas arriver. Cet « impossible à supporter » amène Annabelle à paraître irréprochable dans l'organisation de sa leçon : " j'aurais pas accepté de placer plein de cônes et me dire, ah mais non, c'était pas comme ça que je devais les placer (...) et donc je faisais tout pour paraître organisée, voilà ».

La deuxième raison que nous donne Annabelle pour expliquer l'absence d'écarts entre la préparation et la leçon, correspond au stress généré par le stage, ayant pour conséquence la totale anticipation, de façon à éviter de devoir réfléchir et s'adapter en cours de leçon : «Moi j'avais ma préparation en tête, je donnais la préparation aux élèves (...) et peut-être qu'elles faisaient des erreurs que j'avais pas prévu à la base dans ma préparation et du coup que j'ai pas relevées et que j'ai pas corrigées ». Cette prise de conscience détermine les préoccupations d'Annabelle davantage axées sur la production de l'activité (faire en sorte que la leçon tourne) et le contrôle du savoir à enseigner (la classe suit la leçon). Ces préoccupations constituent deux des trois stades proposés par Maulini (2009) pour décrire l'itinéraire des préoccupations des novices. On peut en déduire qu'Annabelle n'a pas encore atteint le dernier stade du « double contrôle : par le savoir enseigné et appris, l'activité produite et le produit de l'activité » (Maulini, op. cit., p. 68) qui correspond au fait de réguler son enseignement en fonction des réponses et des besoins des élèves, de façon à aboutir sur les apprentissages attendus. 


\section{eJRIEPS 43 janvier 2018}

Qu'il s'agisse du superviseur ou du MS, le fait d'être observé constamment amène Annabelle à ne pas prendre de risques en se conformant aux indications du MS : « J'essayais jamais une toute nouvelle leçon que personne n'avait jamais essayée, qui était sortie de ma tête pendant la nuit, parce que c'était quand même un gros risque et qu'en stage, je prenais pas de risques». De cette façon, elle établit une sorte de «norme de viabilité personnelle »(Ria \& Rouve, 2009, p. 267) constituée de critères personnels comme notamment, l'évaluation du « degré de mise en péril » de sa réussite. Les expériences de stage amènent donc Annabelle à reproduire et non à créer ou expérimenter une autre approche ou d'autres contenus. Ce constat la place donc dans une situation d'inconfort, divisée entre ses conceptions et les SaE proposés en FI et par les MS. Pour expliquer ces tensions émanant du rapport à l'institution, Carnus (2013), repositionne le sujet au cœur d'un système didactique ternaire dans lequel elle place à ses trois sommets le savoir, l'institution dans laquelle le savoir est transposé et converti, et l' « épreuve », au cours de laquelle le savoir est mobilisé. Dès lors, " entre soumission et émancipation, besoin de sécurité et de reconnaissance, le rapport du sujet enseignant à l'institution scolaire apparait ici comme un élément compréhensif voire explicatif du fonctionnement didactique » (Carnus, 2013, p. 31).

\section{Conclusion et perspectives}

Les relations établies entre le sujet, le savoir, l'épreuve et l'institut de formation au sens large (école de formation et école de stage) à partir de l'étude du rapport au savoir, permettent d'apporter des éléments de réponse à la compréhension du système didactique dans lequel se trouve le sujet étudiant-stagiaire et d'en mesurer la complexité. Comme le propose Carnus (2013, p. 32), « intégrer l'analyse de ces relations dans un programme de formation dans des dispositifs spécifiques engageant un travail de subjectivation et de recherche de significations permettrait peut-être d'apaiser le caractère anxiogène de l' "épreuve ", de faire effleurer les tensions, et surtout, de rompre avec l'illusion de la rationalité et de la maîtrise ». Nous pensons que la prise de conscience de la complexité du système didactique et des enjeux qui sous-tendent les relations entre le sujet enseignant, le savoir et l'institut de formation (le rapport au savoir) permet de tenir compte de ces tensions mais aussi des affects et de l'expérience personnelle des étudiants-stagiaires. Pour Terrisse (2003, p. 62), le rapport au savoir peut être considéré comme « un outil d'analyse des difficultés, des obstacles, des avatars mais aussi le témoin de ces acquisitions », en référence à l'apprentissage de savoirs par les 


\section{eJRIEPS 43 janvier 2018}

apprenants, ce qui se révèle être un indicateur précieux à prendre en compte dans la formation.

Notre analyse s'attache à la singularité d'une expérience personnelle s'inscrivant dans un cadre méthodologique que nous postulons générique, applicable pour les autres sujets de notre thèse. Ces premiers résultats nous conforte dans le choix et la pertinence de la méthodologie choisie ainsi qu'en sa rigueur, tant exigée par la méthode clinique (Terrisse, 2013).

Enfin, la mobilisation du cadre de la didactique clinique, à travers cette étude de cas, nous permet de mieux comprendre le fonctionnement du système didactique chez les étudiantsstagiaires par la prise en compte de ce que Ben Jomâa (2015, p. 117) nomme les « implicites du métier d'enseignant : les ressources personnelles du sujet enseignant, sa singularité, son rapport personnel au savoir, son rapport personnel à l'activité, son rapport intime au corps selon l'expertise dont il témoigne ».

Dans le cas d'étudiants-stagiaires, la mise en évidence de l'assujettissement fort à certains MS nous amène à envisager la mise en place d'un travail de collaboration avec les MS en vue d'accompagner les étudiants de façon plus efficace. Cependant, comme le précisent Van Nieuwenhoven et Roland (2015, p. 217) au sujet de la formation des enseignants en Belgique francophone, « il existe peu de balises structurant les rôles attendus des maitres de stage, en haute école comme à l'université. Ceci engendre une impossibilité de cohérence et d'harmonisation des pratiques d'accompagnement et d'évaluation avec les acteurs de l'institut de formation ". Cette absence de balises est également mise en avant par Carlier (2009, p. 83) qui parle d'avantage « d'un manque de structures habilitées à professionnaliser » le dispositif de supervision et d'accompagnement des MS. Même si aucun cadre légal n'existe encore pour l'encadrement des stages, probablement avons-nous un rôle à jouer en tant que formateur, comme l'évoquait Annabelle, dans le fait d'aider l'étudiant à trouver de la cohérence dans son curriculum mais aussi dans la prise en compte du poids de l'assujettissement, tant de l'école de formation que de l'école de stage à travers un dispositif de stage apparemment très contraignant. Ce constat abonde dans le sens de Loizon (2014, p. 45) qui insiste sur l'accompagnement de l'étudiant, tant pour sa formation que pour son métier à venir : « en formation d'enseignant, il nous semble important de comprendre le sujet pour l'aider dans son développement professionnel, l'accompagner afin qu'il trouve de la cohérence dans ses enseignements. Le rôle du formateur pourrait être d'aider le jeune enseignant à clarifier son rapport aux activités physiques en même 


\section{eJRIEPS 43 janvier 2018}

temps que son rapport à l'EPS et à l'institution scolaire, à identifier ses conceptions, à mettre en évidence la dimension éducative de son métier (ses valeurs prioritaires) en complément des savoirs plus techniques qu'il doit enseigner ».

\section{Bibliographie}

Albarello, L. (2011). Choisir l'étude de cas comme méthode de recherche. Bruxelles : De Boeck.

Altet, M. (2000). L'analyse de pratiques. Une démarche de formation professionnalisante ? Recherche et formation, 35, 25-41.

Altet, M. (2003). Les compétences de l'enseignant-professionnel : entre savoirs, schèmes d'action et d'adaptation, le savoir analyser. In L. Paquay, M. Altet, E. Charlier \& P. Perrenoud (Eds.), Former des enseignants professionnels (pp. 27-40). Bruxelles : De Boeck.

Ben Jomâa, H., \& Terrisse, A. (2011). De l'expertise à l'enseignement : analyse comparative du rapport au savoir de deux enseignants d'EPS de spécialités sportives différentes. Education et didactique, 5.3, 61-80. DOI : 10.4000/educationdidactique. 1113

Ben Jomâa, H. (2015). L'implicite du métier d'enseignant: l'expertise personnelle. Un regard didactique clinique de I'EPS. In P. Buznic-Bourgeacq \& L. Gérard, (Eds.), L'apprentissage du métier d'enseignant: constructions implicites, espaces informels et interface de formation (pp. 101-122). Caen : Presse universitaire de Caen.

Blanchard-Laville, C., Beillerot, J., \& Mosconi, N. (1996). Pour une clinique du rapport au savoir. Paris : L'Harmattan.

Bossard, L.-M. (2009). Enseignants débutants : de l'« adolescence professionnelle » à la «post-adolescence professionnelle ». Revue Cliopsy, 2, 65-77.

Buznic-Bourgeacq, P. (2009). La transmission du savoir expérientiel. Etudes de cas et analyses comparatives en didactique clinique de l'EPS (Thèse de doctorat). Université de Toulouse III Paul Sabatier, Toulouse.

Caillot, M. (2014). Les rapports aux savoirs des élèves et des enseignants. In M.-C. Bernard, A. Savard, \& C. Beaucher (Eds.), Le rapport aux savoirs : une clé pour analyser les épistémologies enseignantes et les pratiques de classe (pp. 7-18). 
eJRIEPS 43 janvier 2018

Québec : Livres en ligne du CRIRES. En ligne http://lel.crires.ulaval.ca/public/le_rapport_aux_savoirs.pdf

Carlier, G. (2009). Accompagner et former des maîtres de stage en éducation physique. L'expérience de l'Université catholique de Louvain (Belgique). In J.-F. Desbiens, C. Borges \& C. Spallanzani (Vol. Ed.), Education et francophonie : Vol. $37 \mathrm{n}^{\circ} 1$. La supervision Pédagogique en enseignement de l'éducation physique (pp. 69-88).

Carnus, M.-F. (2009). La décision de l'enseignant en didactique clinique. Etudes de cas en Education Physique et Sportive (EPS). In A.Terrisse \& M.-F. Carnus (Eds), Didactique clinique de l'éducation physique et sportive (EPS). Quels enjeux de savoirs? (pp. 63-81). Bruxelles : De Boeck.

Carnus, M.-F. (2013). Le sujet n'est pas maître dans sa propre institution. In M.-F. Carnus \& A. Terrisse (Eds.), Didactique clinique de l'EPS. Le sujet enseignant en question (pp. 23-32). Paris : Editions EP\&S.

Charlot, B. (1992). Rapport au savoir et rapport à l'école dans deux collèges de banlieue. In Sociétés contemporaines : n¹1-12. Regards sur l'éducation (pp. 119-147).

Charlot, B., Bautier, E., \& Rochex, J.-Y. (1992). Ecole et savoir dans les banlieues... et ailleurs. Paris : Armand Colin.

Charlot, B. (2002). Du rapport au savoir. Eléments pour une théorie. Paris : Anthropos.

Chevallard, Y. (1992). Concepts fondamentaux de la didactique : perspectives apportées par une approche anthropologique. Recherches en didactiques des Mathématiques, 12(1), 73-111.

Cloes, M., Berwart, G., \& Frédéric, O. (2010). Les enseignants en éducation physique wallons connaissent-ils le socioconstructivisme et pensent-ils l'utiliser ? eJRIEPS, 21, 5-25.

Clot, Y. (1995). L'échange avec "un sosie" pour penser l'expérience. Société française, 3 (53), 51-55. En ligne: https://pandor.u-bourgogne.fr/imgviewer/SF/SF_1995_4T_n53/viewer.html?\&ns=SF_1995_4T_n53_001.jpg

Décret définissant la formation initiale des instituteurs et régents : Décret de la Communauté française du 12 décembre 2000 définissant la formation initiale des instituteurs et régents. (2001). Moniteur belge, 19 janvier 2001. En ligne: http://www.gallilex.cfwb.be/document/pdf/25501_000.pdf

Delignières, D., \& Garsault, C. (2004). Libres propos sur l'éducation physique. Paris: Editions Revue EP.S. 


\section{eJRIEPS 43 janvier 2018}

Brossais, E. \& Terrisse, A. (2009). Le poids de l'institution sportive dans l'analyse d'un enseignant débutant-expert en judo. In A. Terrisse \& M.-F. Carnus (Eds), Didactique clinique de l'éducation physique et sportive (EPS). Quels enjeux de savoirs (pp. 63-81) ? Bruxelles : De Boeck.

Filloux, J. (1989). Sur le concept de transfert dans le champ pédagogique. Revue française de pédagogie, 87, 59-75. DOI : 10.3406/rfp.1989.1421

Goigoux, R., Ria, L., \& Toczek-Capelle, M-C. (2009). Mieux connaitre les parcours de formation des enseignants débutants pour mieux les former. In R. Goigoux, L. Ria, \& M.-C. Toczek-Capelle (Eds), Les parcours de formation des enseignants débutants (25-44). Clermont-Ferrand : Presses universitaires Blaise Pascal.

Heuser, F. (2009). Du savoir enseigné et évalué à la référence de l'enseignant d'EPS : étude clinique en Karaté (Thèse de doctorat). Université de Toulouse III Paul Sabatier, Toulouse.

Julien P.-A., Lamonde P., \& Latouche, D. (1975). La méthode des scénarios en prospective. L'Actualité économique, vol. 51 n², 253-281.

Jourdan, I. (2009). Etude du rapport au savoir chez les étudiants en formation initiale en EPS. In A. Terrisse \& M.-F. Carnus (Eds), Didactique clinique de l'éducation physique et sportive (EPS). Quels enjeux de savoirs (pp. 33-48) ? Bruxelles : De Boeck.

Lejeune, C. (2008). Au fil de l'interprétation - L'apport des registres aux logiciels d'analyse Qualitative. Swiss Journal of Sociology, 34 (2), 593-603.

Loizon, D., Margnes, O., \& Terrisse, A. (2008). Analyse des pratiques d'enseignement du judo en EPS. eJRIEPS, 63, 63-82.

Loizon, D. (2009). Les filtres personnels de l'action didactique. Etude de cas dans l'enseignement du judo. In A. Terrisse \& M.-F. Carnus (Eds), Didactique clinique de l'éducation physique et sportive (EPS). Quels enjeux de savoirs (pp. 83-99) ? Bruxelles : De Boeck.

Loizon, D., \& Carnus, M.-F. (2014). L'influence des déterminants personnels dans les choix didactiques des enseignants d'EPS. eJRIEPS, 33, 30-48.

Maulini, O. (2009). Devenir enseignant: invariants et évolution des débuts dans la profession. In R. Goigoux, L. Ria \& M.-C. Toczek-Capelle (Eds), Les parcours de formation des enseignants débutants (pp. 61-78). Clermont-Ferrand: Presses universitaires Blaise Pascal.

Maury, S., \& Caillot, M. (2003). Rapport au savoir et didactiques. Paris : Éditions Fabert. 


\section{eJRIEPS 43 janvier 2018}

Meyer, V. (2008). La méthode des scénarios : un outil d'analyse et d'expertise des formes de communication dans les organisations. Études de communication, 31, 133-156. DOI : 10.4000/edc. 778 .

Mieusset, C. (2013). Les dilemmes d'une pratique d'accompagnement et de conseil en formation. Analyse de l'activité réelle du maître de stage dans l'enseignement secondaire (Thèse de doctorat). Université de Reims Champagne-Ardenne.

Mucchielli, R. (1991). L'analyse de contenu des documents et des communications. Paris : ESF.

Oddone, I., Re, A., \& Briante, G. (1981). Redécouvrir l'expérience ouvrière: vers une autre psychologie du travail ? Paris : Éditions sociales.

Ria, L., \& Rouve, M.-E. (2009). Observatoire du développement professionnel des néotitulaires en collèges « ambition réussite »: trajectoires, activités et identités. In R. Goigoux, L. Ria \& M.-C. Toczek-Capelle (Eds), Les parcours de formation des enseignants débutants (255-269). Clermont-Ferrand: Presses universitaires Blaise Pascal.

Saujat, F., \& Serres, G. (2015). L'activité de l'enseignant d'EPS entre préoccupations et "occupations" : un point de vue développemental. eJRIEPS, 34, 4-30.

Terrisse, A. (2000). La posture clinique en didactique de l'EPS : conditions et conséquences. Grenoble : Communication présentée au $1^{\mathrm{er}}$ colloque de l'ARIS.

Terrisse, A. (2003). Prise en compte du sujet et recherches en didactique des sports de combat : bilan et perspectives. Sciences et motricité, $3 \mathrm{n}^{\circ} 50,55-79$. DOI $10.3917 / \mathrm{sm} .050 .0055$

Terrisse, A. (2008). Le sujet en didactique clinique de l'EPS: Conditions et conséquences pour la recherche. eJRIEPS, 15, 179- 197.

Terrisse, A., \& Carnus, M-F., (2009). Didactique clinique de l'éducation physique et sportive (EPS). Quels enjeux de savoirs ?Bruxelles : De Boeck.

Terrisse, A. (2010). La recherche en didactique clinique sur l'enseignement des sports de combat en EPS: fondements, méthodes et résultats. In La Táctica deportiva y la toma de decisiones. A 'Actes de congressos i jornades' (pp. 1-10). Girona: Universitat. En ligne http://hdl.handle.net/10256/2799

Terrisse, A. (2013). La construction du cas : un enjeu scientifique en didactique clinique de l'EPS. In A. Terrisse \& M.-F. Carnus (Eds), Didactique clinique de l'éducation physique et sportive (EPS). Quels enjeux de savoirs? (125-133). Bruxelles : De Boeck. 


\section{eJRIEPS 43 janvier 2018}

Terrisse, A. (2016). Les analyseurs des pratiques d'enseignement des professeurs d'EPS en didactique clinique. In B. Calmettes, M.-F. Carnus, C. Garcia-Debanc \& A. Terrisse (Eds), didactique et formation des enseignants (423-431). Louvain-LaNeuve: Presses universitaires de Louvain.

Vanlerberghe, G. (2007). La liberté accordée aux élèves par les étudiants en EPS en situation de pré-professionnalisation. Contribution d'un modèle conatif à l'optimisation du curriculum enseignant (Thèse de doctorat). Université du Littoral Côte d'Opale.

Van Nieuwenhoven, C., \& Roland, N. (2015). La formation pratique des enseignants en Belgique francophone. Formation et profession, 23(3), 211-218.

Werthe, C. (1997). Exploration et formalisation de l'expérience professionnelle : l'instruction au sosie. Dialogue, 86. En ligne: http://gfen66.infini.fr/gfen66/IMG/pdf/sosie.pdf

Wittorski, R., \& Briquet-Duhazé, S. (2009). Dynamique de professionnalisation /développement professionnel d'enseignants: étude comparative entre les premiers et seconds degrés. In R. Goigoux, L. Ria \& M.-C. Toczek-Capelle (Eds), Les parcours de formation des enseignants débutants (45-60). Clermont-Ferrand : Presses universitaires Blaise Pascal.

\section{Annexe 1}

\begin{tabular}{|l|l|}
\hline \multicolumn{1}{|c|}{ Scénario guide } & \multicolumn{1}{c|}{ Ce que l'on cherche à identifier } \\
\hline$\rightarrow$ Premier scénario & Dans ce premier scenario, nous cherchons à savoir \\
Celui-ci projette l'étudiant dans le cas extrême d'une & quels sont les cours marquants de la formation (ceux \\
situation dans laquelle la conjoncture économique est & qui présentent suffisamment d'importance, de sens et \\
catastrophique et impose des restrictions budgétaires & de valeur pour être évoqué) et indirectement, ce qui va \\
draconiennes limitant le nombre de cours de la être utile - et utilisé - dans le métier d'enseignant ainsi \\
formation. Concrètement, la question posée consiste à \\
$\begin{array}{l}\text { savoir quels seraient les cours, s'il ne fallait en garder } \\
\text { que deux ou trois, qu'il faudrait conserver à tout dont les étudiants perçoivent la } \\
\text { prix pour devenir un bon professeur d'EP? }\end{array}$
\end{tabular}


Objectif: questionner le rapport identitaire au savoir (selon Charlot, 2002), l'identité professionnelle naissante et le rapport à la formation.

\section{Annexe 2}

\begin{tabular}{|l|l|}
\hline \multicolumn{1}{|c|}{ Scénario guide } & \multicolumn{1}{c|}{ Ce que l'on cherche à identifier } \\
\hline$\rightarrow$ Premier scénario & Dans ce premier scenario, nous cherchons à replacer \\
Un nouveau collègue te parle de sa difficulté à donner & le sujet dans le contexte de sa leçon avec pour objectif \\
une leçon de ... (choisir l'APS enseignée au cours de l' & le remaniement de cette expérience singulière. Il s'agit \\
“épreuve »). Si tu devais l'aider, lui proposerais-tu ta & de saisir le sens que le sujet attribue aux savoirs \\
leçon (celle observée) comme référence ? Si tu lui & théoriques et pratiques (savoirs enseignés, savoirs \\
proposes cette leçon, comment t'y prendrais-tu pour le & pour enseigner, savoirs de la pratique, savoirs sur la \\
convaincre que le contenu de cette leçon correspond & pratique) ainsi que le rapport aux MS et à la \\
bien au contenu à proposer pour une leçon spécifique \\
à cette APS? et quant à l'approche pédagogique, lui \\
préconiserais-tu celle que tu as utilisée pour cette \\
leçon ? enfin, par rapport à la relation didactique avec \\
les élèves, lui conseillerais-tu de procéder de la même \\
façon que tu avais procédé pour cette leçon?
\end{tabular}

\begin{tabular}{|c|c|}
\hline Scénario guide & Ce que l'on cherche à identifier \\
\hline $\begin{array}{l}\rightarrow \text { Second scénario } \\
\text { Ton voisin, élève de classe terminale, te demande } \\
\text { conseil car il veut devenir professeur d'EP. Si tu dois le } \\
\text { conseiller à propos des études et du métier, que lui } \\
\text { dirais-tu? Que lui dirais-tu à propos de la formation? } \\
\text { Que va-t-elle lui apporter? Et les stages, que vont-ils } \\
\text { lui apporter de plus, de différent, de complémentaire } \\
\text { par rapport aux cours? A quoi doit-il s'attendre comme } \\
\text { difficultés, comme découverte positive, comme } \\
\text { enrichissement, comme déception? }\end{array}$ & $\begin{array}{l}\text { Dans ce second scénario, nous cherchons à accéder à } \\
\text { la signification que prend pour lui, après-coup, la FI } \\
\text { tant sur le plan du développement professionnel, qu'au } \\
\text { niveau des curriculums de formation : } \\
\text { - cours marquants } \\
\text { - place des stages dans le dispositif de } \\
\text { formation et dans l'acquisition des savoirs. } \\
\text { Objectif : questionner le rapport à la FI. }\end{array}$ \\
\hline
\end{tabular}

